\title{
Estudio evolutivo de las asignaturas del Núcleo de Formación General y de Instrumentalidades del Currículo Pleno del Curso de Biblioteconomía de la Facultad de Filosofía y Ciencias de la UNESP - Marília
}

Evolutive study of the discipline of the General Formation Nucleus and Instrumentalities of the Full Curriculim of the Librarianship Course of the Faculdade de Filosofia e Ciências da Unesp - Marilia.

\author{
Ely Francina Tannuri de Oliveira (1), João Batista Ernesto de Moraes (2) \\ y Maura Duarte Moreira Guarido (3)
}

(1) Faculdade de Filosofia e Ciências - Unesp, - Av. Higinio Muzzi Filho, 737 Marília - São Paulo Brasil - CEP: 17525-900, etannuri@flash.tv.br. (2) jota@marilia.unesp.br. (3) mauraguarido@gmail.com

\begin{abstract}
Resumen
Se analizan retrospectivamente los diferentes currículos del Curso de Biblioteconomía de la Facultad de Filosofía y Ciencias (FFC), de Unesp Marília, desde el primero de ellos, basado en el Currículo Mínimo establecido por el Ministerio de Educación en 1962, hasta el más reciente, de 2003, basado en las Directrices Curriculares Nacionales, establecidas por la Ley de Directrices y Bases de 2001, teniendo como enfoque el estudio de las materias llamadas inicialmente de instrumentales, y que hoy forman parte del Núcleo de Formación General e Instrumentalidades. Se justifica este abordaje por el hecho de que las investigaciones sobre currículos privilegian las materias de Formación Específica. Se pretendió, de esta forma, plantear una evolución histórica del Currículo del Curso de Biblioteconomía de la FFC-UnespMarília, desde el prisma del Núcleo de Formación General e Instrumental, para evaluar cómo estas asignaturas contribuyen a la formación del profesional bibliotecario en los diferentes enfoques asumidos en las reformulaciones curriculares.
\end{abstract}

Palabras-Clave: Biblioteconomía. Currículo. Enseñanza de Biblioteconomía. Asignaturas instrumentales. Asignaturas de formación general.

\section{Introducción}

En 1959, el Ministerio del Trabajo reconoció la profesión de Bibliotecario dentro del cuadro de las profesiones liberales. Además, frente a la exigencia de la Ley de Directrices y Bases (1961), de que el Consejo Federal de Educación fijase el currículo mínimo de los cursos superiores, fue aprobado en 1962 el currículo mínimo

\begin{abstract}
The history of the curricula of the Librarianship Course of the Faculdade de Filosofia e Ciências (FFC) of the Unesp Marilia is analyzed, beginning with the first of them, that was based on the Minimum Curriculum established by the Ministry of the Education in 1962, until the most recent, of 2003 , based in the Diretrizes Curriculares Nacionais, established in the "Diretrizes e Bases" Law of 2001, having as a focus the study of the so-called "instrumental" subjects, that are today part of the Nucleus of General and Instrumental disciplines. Usually, curricula research is focused in the specific subjects, letting apart the generic competences. This paper evaluates how these disciplines has contributed to the education of the professional librarian in the different curricular reformularizations.
\end{abstract}

Keywords: Librarianship. Full curriculum. Librarianship teaching. Instrumental disciplines. General formation disciplines.

para el curso de Biblioteconomía, quedándose así consignado el curso en nivel superior, con tres años de duración. Con fuerte influencia norteamericana y con superdimensionamiento de las asignaturas técnicas, el curso de Biblioteconomía se proponía, entonces, a formar el profesional destinado a organizar, conservar, clasificar y catalogar acervos; en fin, un profesional "eficiente" y "organizado", apto a usar 
normas y tablas, pero incapaz de atender al usuario, que se tornaba más exigente.

El poco énfasis dado a las asignaturas dirigidas a las humanidades, para el social y para la investigación caracterizó ese primer currículo de nivel superior, impregnado de la ideología impuesta a toda enseñanza superior brasileña: se exigía del futuro profesional gran eficiencia y capacidad de ejecutar servicios, pero la investigación era irrelevante.

Las asignaturas dirigidas a la investigación no estaban presentes en el currículo, porque tal práctica era inexistente en el área. Sin embargo, desde mediados de la década de 70 , con la creación del primer Curso de Postgrado, la investigación en el área se torna un hecho, y encuentra reflejos en el establecimiento de asignaturas del Currículo Mínimo que contemplen este nuevo campo.

En este estudio, se buscó analizar los diferentes currículos del Curso de Biblioteconomía de la Facultad de Filosofía y Ciencias (FFC), de la Unesp Marília, desde el primero de ellos, que se basaba en el Currículo Mínimo establecido por el Ministerio de la Educación (MEC) en 1962, hasta el más reciente, de 2003, basado en las Directrices Curriculares Nacionales, establecidas por la Ley de Directrices y Bases (LDB) de 2001 , teniendo como enfoque el estudio de las materias llamadas inicialmente instrumentales, y que hoy son parte de los Núcleos de Formación General e Instrumentalidades, nominadamente Elementos de Lógica para Documentación, Métodos Cuantitativos Aplicados a la Ciencia de la Información, Expresión Escrita en Lengua Portuguesa, Literaturas de Lengua Portuguesa y Lingüística y Documentación.

Se justifica este abordaje por el hecho de que las investigaciones sobre currículos privilegian las materias de Formación Específica, así como por el hecho de que los autores imparten las asignaturas arriba listadas. Se pretendió, de esta forma, trazar una evolución histórica del Currículo del Curso de Biblioteconomía de la FFC-Unesp-Marília, desde el prisma del Núcleo de Formación General y de Instrumentalidad, para que evaluen cómo estas asignaturas contribuyen para la formación del profesional bibliotecario en los diferentes enfoques asumidos en las reformulaciones curriculares.

Como procedimiento de trabajo, a través del análisis documental, se optó por comparar los diferentes currículos: el Currículo Pleno de 1977 (fundamentado en el Currículo Mínimo de 1962); el Currículo Pleno de 1984 (fundamentado en el Currículo Mínimo de 1982); el Currículo Pleno de 1989 (fundamentado en el Currículo Mínimo de 1982); el Currículo Pleno de 1997 (funda- mentado en el Currículo Mínimo de 1982); y el Currículo Pleno de 2003 (fundamentado en las Directrices Curriculares de 2001).

Procedente de la creación de la UNESPUniversidad Estadual Paulista, el 30 de enero de 1976, el curso de Biblioteconomía inició sus actividades en el campus de Marília, el 06 de abril de 1977, reconocido por el MEC conforme Decreto $n^{\circ} 145$, de 11/02/81, publicado en el Diário Oficial da União (DOU) de 13 de febrero de 1981, coincidiendo con la creación de la propia universidad.

\section{Contextualización histórica}

El Campus de Marília comprende la Facultad de Filosofía y Ciencias que, además de los cursos de Biblioteconomía y Archivología, ofrece los cursos de licenciatura en Pedagogía, Ciencias Sociales, Filosofía, Relaciones Internacionales, Fisioterapia, Terapia Ocupacional y Fonoaudiología, así como un curso de postgrado, en nivel de maestría y doctorado, en Ciencia de la Información, Educación, Ciencias Sociales, y Maestría en Filosofía.

El período de 1977 a 1983 vigoró una primera estructura curricular, conforme Resolución UNESP $n^{\circ}$. 18, de 11 de abril de 1978, publicada en el $D O$. de 27/04/78.

En aquel momento, el curso vino al encuentro de una realidad que se imponía: la creación e instalación, en Marília, de la Biblioteca Central de la Red de Bibliotecas de la UNESP.

De ese modo, el énfasis inicial del curso se centró más en la formación de un profesional bibliotecario para bibliotecas universitarias, en el cual actividades de formación de acervos y diseminación de informaciones, en el ámbito académico, adquirían especial importancia.

Tal abordaje se daba, en aquel momento, en consonancia con una visión de la profesión de los años 70 cuando, pasada más de una década de reconocimiento de la profesión y del establecimiento del primer currículo mínimo para los cursos universitarios del área, se intentaba romper con la influencia tecnicista norteamericana que norteó la formación del área entre los años 30 y 60.

En 1982 es establecido el nuevo currículo mínimo de la profesión, preocupado por integrar la concepción humanista francesa (bajo la influencia de la École de Chartres) -que norteó la enseñanza en la década de 30 - con la concepción técnica norteamericana que se preocupaba esencialmente con la cuestión del usuario. 
Así, en 1984 el curso de Biblioteconomía de la UNESP implantaba su segundo currículo pleno, aprobado por la Resolución UNESP $\mathrm{n}^{\circ}$. 30, de 04 de junio de 1984, publicada en el DOU de 05/06/84.

En 1988, tras constantes evaluaciones curriculares, el Departamento de Biblioteconomía elaboró propuesta de alteración de esa nueva estructura curricular incluyendo la añadidura y disminución de créditos, así como modificaciones en la seriación y nomenclatura de algunas asignaturas sin, no obstante, alterar la estructura obligatoria aprobada por el CFE/MEC. Aprobado por la resolución UNESP 4/88 el nuevo currículo pasó a implantarse a partir de 1989.

En esta época, el cuerpo docente todavía se organizaba con pocos profesores titulados. La gran mayoría todavía iniciaba la maestría, buscando en los cursos de la Universidad de São Paulo la capacitación necesaria. Tres hechos caracterizaron estos primeros tiempos del curso:

- la búsqueda de capacitación de los docentes,

- el incentivo de las actividades de investigación en la licenciatura,

- la búsqueda de una política e implementación curricular en bases sólidas para que se pudiera configurar el profesional que venía siendo formado por el curso.

Se realizaron, en esa época, varias evaluaciones curriculares. En 1986, la ABEBDAsociación Brasileña de Enseñanza de Biblioteconomía y Documentación -entidad creada en 1967 con el objetivo de coordinar las escuelas de Biblioteconomía del país- promovió un conjunto de discusiones con el objetivo de evaluar el entonces nuevo currículo de Biblioteconomía. En ese sentido, los ENEBCls-Encuentros $\mathrm{Na}$ cionales de Enseñanza de Biblioteconomía y Ciencia de la Información realizados en Recife en 1986 y en Brasília en 1989 propiciaron un amplio debate sobre cuestiones como la estructura curricular, salidas profesionales, prácticas y mercado de trabajo. En el ámbito del curso, se promovieron debates y reuniones, en los cuales se acordó investigar la situación en profundidad. Los resultados fueron analizados para inferir cómo se podría mejorar la calidad del curso delinenado claramente el profesional formado por el curso.

Paralelamente, fueron creados 5 Grupos de Regionales de Estudios Curriculares en Biblioteconomía. El curso de Biblioteconomía de la UNESP-Marília formó parte del grupo São Paulo y realizó en el período de 1989 a 1992 estudios de evaluación curricular. A pesar de que el Departamento de Biblioteconomía estaba en pro- ceso de titulación de su cuerpo docente, participó activamente en el grupo de estudios curriculares de São Paulo, así como de la gestión administrativa de la ABEBD durante dos mandatos consecutivos (1991-1995).

Ya en esa época la investigación, como iniciación científica del discente, adquiría carácter relevante y daba prenuncios de constituirse en el núcleo de las actividades académicas.

En 1992, el II Encuentro Nacional de enseñanza de la Biblioteconomía y Ciencia de la Información, recomendó la realización de proyectos pedagógicos como apoyo para la política educacional en Biblioteconomía.

Con la instalación de los Consejos de Curso (1990) en los cursos de licenciatura de la UNESP, se inició un trabajo de construcción del Proyecto Pedagógico del Curso de Biblioteconomía. Inicialmente este proyecto fue reconocido en 1994.

El creciente y significativo interés demostrado por la comunidad discente y docente en lo que hace referencia a la estructura curricular del curso desencadenó una nueva reestructuración. Entre agosto de 1994 y abril de 1996, se realizaron una serie de discusiones, análisis, estudios y diagnósticos del curso que acabaron por transformarse en una nueva y profunda reestructuración curricular, centrada, por lo tanto, en cuestiones de ámbito nacional -las recomendaciones curriculares de la ABEBD.

Dentro de la política académica del entonces Departamento de Biblioteconomía y Documentación (y actualmente Departamento de Ciencia de la Información) fue implantada, en 1997, una nueva estructura curricular del curso de Licenciatura en Biblioteconomía, fruto del nuevo Proyecto Pedagógico para el curso, en el cual se registraron tres años de discusiones que englobaron los segmentos docente y discente del curso, buscando propiciar una formación que permita al licenciado en Biblioteconomía actuar como un efectivo profesional de la información al lado de otras profesiones de la información.

Tales discusiones estuvieron, primordialmente, dirigidas al delineamiento de una concepción curricular en la cual la malla se viera no como un fin en sí misma, sino como un instrumento para concretizar una filosofía de enseñanza.

Como resultado de tal concepción, fue propuesto e implantado un currículo pleno de Biblioteconomía que fue operacionalizando en estrecha conexión con las líneas de investigación departamentales. 
A lo largo de los seis años de pervivencia del nuevo currículo del curso, lo que se pudo observar fue una preocupación cada vez más destacada por abordar la información a partir de una concepción macro, en la que los distintos entornos llevan a procedimientos distintos, pero complementarios.

Con la aprobación de la Ley no. 9394/96 — Ley de Directrices y Bases de la Educación Nacional- la universidad pasa a tener competencia para fijar los currículos de sus cursos y programas, siempre que sean observadas las directrices generales, creando así la posibilidad de flexibilidad y adaptaciones curriculares, que puedan ser hechas tanto en nivel regional, como nacional e internacional. En esta ley, el currículo mínimo dejó de existir, y fue substituido por directrices que nortearon la formación, dejando al mismo tiempo espacio para flexibilización curricular en el ámbito nacional y regional, ampliándose el abanico de posibilidades de actuación profesional.

A partir de esta nueva determinación legal, y en función de los ajustes que se vieron necesarios en el currículo en vista de las constantes evaluaciones curriculares realizadas, se justificó la propuesta de reestructuración curricular del curso de Biblioteconomía de la UNESP-Marília, realizada el año de 2003.

\section{Evolución de las asignaturas Instrumentales}

\subsection{Métodos Cuantitativos}

La preocupación por la inclusión de la Metodología de la Investigación nació a partir del momento en que se inició la investigación en el área. Sin embargo, la aparición como materia obligatoria sólo ocurre en el Currículo Mínimo de 1982, teniendo como desdoblamiento Métodos Cuantitativos Aplicados a la Biblioteconomía, o sea, Estadística y Bibliometría. La propia materia Metodología de la Investigación no nexistía en el Currículo Mínimo establecido por la CFE en 1962. Además, la desarticulación de contenidos creaba lagunas en los currículos. Kohler y Toledo registran la existencia, ya en esta época, de un curso cuyo currículo contenía Métodos Cuantitativos Aplicados a la Biblioteconomía, asignatura que requiere conocimientos de investigación, y, sin embargo, se suprimió del mismo la asignatura Metodología de la Investigación.

El debate y las críticas con respecto al currículo de Biblioteconomía se ampliaron considerablemente en los años 70. La desarticulación curricular era frecuentemente resaltada en las evaluaciones curriculares. No obstante, aunque la conciencia de las fallas era mayor que las suge- rencias para allanarlas, una de las propuestas que comenzaron a hacerse frecuentes se refería a la necesidad de ofrecerse al alumno un fundamento metodológico para la investigación y para el desempeño de sus funciones profesionales y de instrumentarlo para el trato con cuantificaciones. En este sentido, Figueiredo (1977, p. 260), en el $9^{\circ}$. Congreso Brasileño de Biblioteconomía y Documentación, realizado en 1977, criticó la inadecuación del currículo, enfatizando,

la necesidad de preparar bibliotecarios para las tareas de investigación, dándoles nociones de métodos científicos y de estadística para poder contribuir con soluciones creativas para los problemas de administración y evaluación, así como para los de organización, control y diseminación de la información.

A pesar de las críticas y controversias, al final de la década de 1970, ya constaban en los currículos plenos de algunos programas algunas de las asignaturas de "análisis cuantitativo". Se fortalece el movimiento para el cambio de currículo, y la Estadística se convierte en contenido obligatorio en el nuevo Currículo Mínimo, insertado en la materia Metodología de la Investigación; ésta, a su vez, contemplada entre las Materias Instrumentales.

De esta forma, la combinación de varios factores fue responsable de la inclusión de las asignaturas Estadística y Bibliometría, esto es, de asignaturas para el análisis cuantitativo, en los currículos, resaltándose entre ellos, el desarrollo de la investigación en el área con la utilización del paradigma dominante en las ciencias sociales, la expansión de los cursos y la creación y desarrollo de las asociaciones y entidades profesionales.

\subsection{Lógica}

En el currículo mínimo de Biblioteconomía aprobado por el Consejo Federal de Educación en 1982, se prevé la Lógica como materia instrumental en los cursos de licenciatura del área de Biblioteconomía. Definida como acto de pensar, abarca cuestiones específicas como la percepción, el juicio y el raciocinio, la inducción y la deducción, entre otros aspectos.

Guimarães (2003) afirma que es "hecho consensual que la Lógica posee papel importante en la enseñanza del área de Organización de la Información/Análisis Documental (Clasificación, Indización, Resúmenes, etc.), al revelar una instrumentalidad que remonta a los principios de los sistemas de clasificación". Con el desarrollo científico e industrial a mediados del siglo XIX, se pasó a discutir la posibilidad de cambios en los sistemas, como el agrupamiento o separación de 
asignaturas, con la consecuente modificación en los sistemas jerárquicos (Guimarães, 2003).

Los sistemas jerárquicos de clasificación continúan siendo, en la actualidad, la mejor herramienta para la organización del conocimiento. Los análisis de esos sistemas, que representan todo el conocimiento, presentan variaciones en la cantidad, cualidad y tipología de los documentos generados y reflejan como las unidades de información trabajan esas diferencias para la conservación y diseminación de la información de manera aceptable. Esas unidades de información utilizan varios tipos de lenguajes documentales para enfrentar esa nueva realidad, pues los cambios son veloces, y la rapidez de raciocinio es necesaria para que los usuarios puedan ser atendidos satisfactoriamente.

Considerando que la Lógica como ciencia se encuentra bien lejos de las preocupaciones de los bibliotecarios, su inclusión se justifica porque el estudio de la lógica en clasificación es esencial, pues la lógica es un lenguaje que se caracteriza como formal, y porque, al utilizarse del análisis documental, el profesional bibliotecario necesita ejecutar una serie de procedimientos lógicos, que se resumen en la identificación y estructuración, a través de instrumentos específicos, de las llamadas informaciones significativas o relevantes. En ese instante surge la operación del uso de la lógica, porque están implícitas ahí las relaciones de inclusión/exclusión, todo/parte, género/especie, que están fundamentados en juicios y razonamientos.

Es a partir de ese momento que el profesional tiene la capacidad de programar la traducción del contenido del texto en etapas secuenciales lógicas. A partir del análisis documental, se establece la identificación de los conceptos dentro de un sistema bibliográfico preescogido para la determinación del tema y su respectiva representación. Una vez determinados los conceptos y sus identificaciones $y$, teniendo como meta el tema, el clasificador parte del uso de las Leyes de la Lógica Dialéctica para determinar la coordinación y subordinación de esos conceptos, pues es cuando se ejecuta la transición del concepto mayor al menor (subordinación lógica o relación vertical). Esta ley se define como relación jerárquica, basada en la identidad parcial de los conjuntos de características de los conceptos superordenados y subordinados implicados. Al apoyarse en la lógica, todos los sistemas de clasificación tienen como finalidad eliminar falsas interpretaciones de sus reglas; el ideal de cada sistema creado es la comunicación unívoca, sin ambigüedad, con una interpretación única.
3.3. Lengua Portuguesa y Literaturas de Lengua Portuguesa y Historia de la Literatura

Durante casi veinte años, desde la implantación del curso en 1977 hasta la reestructuración de 1997, las asignaturas Lengua Portuguesa y Literaturas de Lengua Portuguesa e Historia de la Literatura permanecieron con la misma denominación. Este hecho puede sugerir, también, que su enfoque haya permanecido inalterado durante todo ese tiempo, sin que cambiara en la reestructuración curricular de 1984, ni en la alteración y adaptación de 1989. A partir de 1997, queda clara la adopción en ambas un nuevo abordaje, separándose los aspectos relativos a los estudios de la Lengua Portuguesa, principalmente la expresión escrita, de aquéllos relacionados con la interfaz Lingüística-Ciencia de la Información; y fundiéndose los aspectos de estudio de la literatura en apenas una asignatura (Literaturas de Lengua Portuguesa), restringiéndose el abordaje anterior, con un carácter más amplio, a aspectos más dirigidos a la literatura nacional y lusitana.

Estos enfoques tienden a reforzar el carácter interdisciplinar de la Ciencia de la Información, así como a destacar los lazos con la Lingüística. Además, surge una aproximación a la asignatura Análisis Documental, también impartida en el mismo semestre del primer año del Curso de Biblioteconomía, con el objetivo de suministrar las bases lingüísticas necesarias para las operaciones de análisis y síntesis documental. Por fin, se debe destacar la conexión de la asignatura Lingüística y Documentación con las asignaturas Elementos Instrumentales en Análisis Documental y La interfaz Análisis Documental, Lingüística Documental y Terminología ofrecidas en el Curso de Postgrado.

Se debe destacar, también, que la asignatura Expresión Escrita en Lengua Portuguesa, según consta en el Plan de Enseñanza, tiene por objetivo capacitar a los alumnos para la redacción de textos científicos, una vez que, a partir del tercer año, deberán desarrollar el proyecto y la redacción de la tesina final, lo que refuerza el carácter instrumental de la asignatura en la formación del futuro bibliotecario.

\section{Análisis comparativo}

En la tabla I, se puede visualizar la evolución de las tres asignaturas en cuestión, a través de las diversas reformulaciones curriculares: Se puede observar que las tres asignaturas fueron contextualizándose con relación al Curso de Biblioteconomía, tornándose cada vez más aplicadas, sin perder, no obstante, el carácter de formación general o instrumentalidad. 


\begin{tabular}{|c|c|c|c|c|c|}
\hline & Currículo Pleno de 1977 & Currículo Pleno de 1984 & Currículo Pleno de 1989 & Currículo Pleno de 1997 & Currículo Pleno de 2003 \\
\hline \multirow{3}{*}{$\begin{array}{l}\frac{0}{4} \\
\frac{1}{2} \\
\frac{1}{2} \\
\frac{0}{0} \\
\frac{0}{4}\end{array}$} & $\begin{array}{l}\text { Matemáticas } \\
\text { Estadística }\end{array}$ & $\begin{array}{l}\text { Matemáticas } \\
\text { Estadística }\end{array}$ & $\begin{array}{l}\text { Matemáticas } \\
\text { Estadística }\end{array}$ & $\begin{array}{c}\text { Matemáticas } \\
\text { Estadística aplicada a la } \\
\text { Biblioteconomía }\end{array}$ & $\begin{array}{l}\text { Métodos Cuantitativos } \\
\text { Aplicados a la Ciencia de } \\
\text { la Información }\end{array}$ \\
\hline & Lógica & Lógica & Lógica & $\begin{array}{l}\text { Elementos de Lógica } \\
\text { para Documentación }\end{array}$ & $\begin{array}{l}\text { Elementos de Lógica } \\
\text { para Documentación }\end{array}$ \\
\hline & $\begin{array}{l}\text { Lengua Portuguesa y } \\
\text { Literaturas de Lengua } \\
\text { Portuguesa } \\
\text { Historia de la Literatura }\end{array}$ & $\begin{array}{l}\text { Lengua Portuguesa y } \\
\text { Literaturas de Lengua } \\
\text { Portuguesa } \\
\text { Historia de la Literatura }\end{array}$ & $\begin{array}{l}\text { Lengua Portuguesa y } \\
\text { Literaturas de Lengua } \\
\text { Portuguesa } \\
\text { Historia de la Literatura }\end{array}$ & $\begin{array}{l}\text { Expresión Escrita en } \\
\text { Lengua Portuguesa } \\
\text { Lingüística } \\
\text { y Documentación } \\
\text { Literaturas de Lengua } \\
\text { Portuguesa }\end{array}$ & $\begin{array}{l}\text { Expresión Escrita en } \\
\text { Lengua Portuguesa } \\
\text { Lingüística } \\
\text { y Documentación } \\
\text { Literaturas de Lengua } \\
\text { Portuguesa }\end{array}$ \\
\hline
\end{tabular}

Tabla I. Evolución de los currículos plenos desde 1977 a 2003.

Se observa, todavía, que la cuestión de la investigación permea las asignaturas, al proveer elementos para la identificación y el estudio de nuevas cuestiones en el área.

\section{Consideraciones finales}

El estudio reveló que las materias objeto de este estudio siempre estuvieron presentes en todos los currículos, aunque desdobladas en asignaturas con énfasis diferentes. De esta forma, la materia Lengua Portuguesa y Literaturas de Lengua Portuguesa, a través de las reformulaciones curriculares, se desdobló en tres asignaturas: Expresión Escrita en Lengua Portuguesa; Lingüística y Documentación; y Literaturas de Lengua Portuguesa. La asignatura Lógica pasó a denominarse Elementos de Lógica para Documentación. Las asignaturas Matemáticas y Estadística pasaron a incorporar la asignatura Métodos Cuantitativos Aplicados a la Ciencia de la Información. Así, se enfatizó el aspecto instrumental de esas asignaturas que pasaron a constituirse, realmente, en instrumentos para generar conocimientos en el área de Ciencia de la Información. Véase el caso de la materia Lengua Portuguesa y Literaturas de Lengua Portuguesa: al optarse por el desdoblamiento en tres asignaturas, se privilegió el estudio de la lengua con fines de redacción de textos científicos (Expresión Escrita en Lengua Portuguesa), el estudio de aspectos de interfaz entre la Ciencia de la Información y la Lingüística (Lingüística y Documentación) y el refuerzo de elementos de formación cultural (Literaturas de Lengua Portuguesa). Se sugiere que estas asignaturas, debido a su carácter general e instrumental, continúen evolucionando a través de las varias reformulaciones curriculares.

\section{Referencias}

Bertachini, Maria de Lurdes; Guimarães, José Augusto Chaves; Vidotti, Silvana Aparecida Borsetti Gregório. Anteprojeto de reformulação curricular do curso de Biblioteconomia da UNESP. Marília: FFC - UNESP, 1994.

Figueiredo, N. M. Currículo de Biblioteconomia: uma questão de mudança de orientação. // Congresso brasileiro de biblioteconomia e documenação, 9, Jornada Sul Rio Grandense de Biblioteconomia, 5, 1977, Porto Alegre. Anais...Porto Alegre, 1977.

Guimarães. J. A. C. Moderno profissional da informação: elementos para sua formação no Brasil. // Transinformação. Campinas. 9:1 (jan./abr. 1997) 124-137.

Guimarães, J. A. C. O caráter instrumental da Lógica no ensino de organização da informação/ análise documentária em cursos de Biblioteconomia, 2003. Disponível em: http://www.ofaj.com.br, em 27 de maio de 2007.

Mueller, S. P. M. O ensino de biblioteconomia no Brasil. UU Ciência da Informação. Brasília. 14:1 (jan./jun, 1985) 315.

Oliveira, E. F. T. As disciplinas instrumentais para análises quantitativas no currículo do curso de graduação em Biblioteconomia da Unesp - Marília. Dissertação (mestrado) Universidade Estadual Paulista "Júlio de Mesquita Filho". Marília: Faculdade de Filosofia e Ciências, 1995.

Población, D. A. (coord.) Ensino de graduação em biblioteconomia no Estado de São Paulo. São Paulo: ECA USP, 1992.

Venturini, G. Estruturas curriculares do curso de biblioteconomia da Unesp: elementos para uma análise comparativa de conteúdos de formação profissional. TCC (Trabalho de Conclusão de Curso) Faculdade de Filosofia e Ciências, Marília, 1999. 